\title{
NIVELES Y DISTRIBUCIÓN GEOGRÁFICA DE LA COBERTURA SANITARIA EN ESPAÑA
}

\author{
POR \\ GLORIA FERNÁNDEZ-MAYORALAS \\ y VICENTE RODRÍGUEZ
}

\section{Introducción}

A partir de la promulgación de la Ley General de Sanidad de 1986 (BOE 102, de 29 de abril, 1986) se da carta de naturaleza, de forma explícita, a la universalización de la cobertura sanitaria entre la población, idea que ya estaba presente en la sanidad española en los años anteriores y que se vertebra a partir de ese año en el Sistema Nacional de Salud que tiene a la Seguridad Social como su principal soporte, incluyendo a otras modalidades públicas (mutualidades y beneficencia). Fuera de este esquema se encuentra la iniciativa privada como instrumento «complementario» de cobertura sanitaria.

Esta universalización se puede analizar a través de las cifras aportadas por diversos estudios e informes. Las metodologías para la obtención de estos resultados no son estrictamente comparables -ya sean datos objetivos, p.e. extraídos de las Memorias del Instituto Nacional de la Salud (Jimeno Martínez, V., 1982); subjetivos, provenientes de encuestas de opinión (Ministerio de Sanidad y Consumo, 1987; CuAnter, 1989; Ministerio de Sanidad y Consumo, 1993); o informes oficiales (World Health Organization, 1981; World Health Organiza-

Gloria Fernández-Mayoralas y Vicente Rodríguez. Instituto de Economía y Geografía. CSIC. Madrid 
tion, 1991)-, ni distinguen entre una cobertura total de la población (que incluye los solapamientos o situaciones de cobertura múltiple) y otra referida exclusivamente a la cobertura de cada modalidad. A pesar de ello, todos estos estudios afirman que en torno al $95 \%$ de la población goza actualmente de una cobertura sanitaria de carácter público.

A efectos de profundizar en el análisis de la cobertura sanitaria, conviene diferenciar precisamente entre estos dos tipos de cobertura, la total y la exclusiva, señalando de forma clara los solapamientos o coberturas múltiples que se produzcan y distinguiendo entre las modalidades de carácter público en su financiación: la Seguridad Social (SS), la Beneficencia (BEN) y las Mutualidades del Estado (acogidas a la Seguridad Social -MSS- o a Seguros Privados -MSP-), de aquéllas otras con financiación privada: los Seguros Médicos Privados (SMP), los Concertados de Empresa (SCE), las Igualas Médicas (IGU) o el recurso al Médico Privado (MP) en sentido estricto.

Otro de los objetivos fundamentales es identificar los modelos geográficos que se perfilen en la distribución de la cobertura sanitaria en sus modalidades más extendidas.

\section{Metodología}

La Encuesta Nacional de Salud de 1993 (en adelante ENSE93) permite el análisis de la cobertura sanitaria a partir de los tres conceptos citados: cobertura total, cobertura exclusiva y solapamiento de cobertura, que han requerido procesos de cálculo más complejos que los que ofrecía el análisis estadístico estándar (Rodríguez, V.; Fernández-Mayoralas, G.; Pumares, P. y Barrios, L., 1994).

La pregunta sobre cobertura sanitaria en los cuestionarios infantil (0 a 15 años) y de adultos (16 y más años) de la ENSE93 faculta la obtención de respuestas múltiples (hasta cuatro posibles), según que el encuestado sea titular o beneficiario de una o más modalidades de seguro, público o privado, o que responda a ello en primer o segundo lugar, de tal forma que la frecuencia obtenida es superior al $100 \%$ de personas encuestadas. Así, en cobertura total se considera todo entrevistado que responda ser, como titular o beneficiario, detentador de una modalidad de seguro cualquiera, bien como primera 
o segunda respuesta. Estará cubierto de forma total por dicho seguro, pero a la vez, quizás, por otro, con tal de que así responda en cualquiera de sus posibilidades.

El cálculo de la cobertura sanitaria total obliga a generar variables ficticias, para cada una de las modalidades, en las que acumular aquellos individuos con una modalidad cualquiera. Los valores totales de cada modalidad de seguro se obtienen mediante el procedimiento MULT RESPONSE del programa estadístico SPSS-X.

La cobertura exclusiva, en cambio, pretende identificar al individuo cuando, en cualquiera de sus cuatro posibilidades, está cubierto únicamente por una modalidad de seguro. De esta forma toda persona que cumple esa condición aparecerá una sola vez, en contraposición con otros individuos que tienen coberturas múltiples. Para el proceso de cálculo es necesario que se responda en cualquiera de sus cuatro posibilidades (titular o beneficiario, primera o segunda respuesta) a una modalidad, pero no al resto. Para ello también se generan variables ficticias que identifiquen estas condiciones, que luego son acumuladas para extraer el número de individuos por medio del programa SPSS-X.

Se han hallado los intervalos de confianza que acompañan a los valores absolutos con objeto de dotarlos de la oscilación adecuada según las características de la muestra. Se ha aplicado el modelo propuesto por Quesenberry y Hurst para el cálculo de los intervalos de confianza para las modalidades de cobertura, según la fórmula:

$$
I_{i}^{Q H}=\frac{2 Y_{i}+c \pm\left(c\left[c+\frac{4 Y_{i}\left(n-Y_{i}\right)}{n}\right]\right)^{\frac{1}{2}}}{2(n+c)}
$$

donde $\mathbf{n}$ es el tamaño de la muestra, $\mathbf{c}$ es $X^{2}{ }_{a, \mathrm{t}-1}$, siendo $\alpha$ el nivel de confianza, fijado en este caso en el $95 \%$, $\mathbf{t}$ cada una de las modalidades de cobertura y $\mathbf{p}_{1} \ldots, \mathbf{p}_{\mathrm{t}}$ las probabilidades de pertenecer a una modalidad determinada.

A partir de la cobertura total y exclusiva se define el concepto de solapamiento que describe la situación de los individuos que son detentadores de varias modalidades al mismo tiempo, como titulares o beneficiarios. Para ello ha sido necesario crear un vector por cada entrevistado y una matriz que recoge todas las duplicidades de co- 
bertura. Este proceso de cálculo ha requerido de un programa FORTRAN, creado a tal efecto (Rodríguez, V.; Fernández-Mayoralas, G.; Pumares, P. y Barrios, L., 1994).

\section{Cobertura Sanitaria Total}

El primer hecho a destacar es la clara contraposición entre las cifras de cobertura provista por modalidades de carácter público (Seguridad Social, Mutualidades y Beneficiencia) y aquéllas relativas a modalidades privadas (Seguros Médicos Privados, Concertados de Empresa, Médico Privado en sentido estricto e Igualatorios) (Cuadro I). Así, tanto en la población adulta como en la infantil, el conjunto de la cobertura pública representa el 98,5\%, mientras que las modalidades privadas, en su totalidad, alcanzan el $8,5 \%$ para los adultos y el 6,8 \% para los menores de 16 años. Estos datos están en consonan-

CUADRo I

COBERTURA TOTAL

\begin{tabular}{|c|c|c|c|c|c|c|}
\hline Modalidades & $\begin{array}{c}\text { Pob. } \\
\text { adulta } \\
(\%)\end{array}$ & $\mathbf{I}_{-}$ & $\mathbf{I}^{+}$ & $\begin{array}{c}\text { Pob. } \\
\text { infantil } \\
(\%)\end{array}$ & $\mathbf{I}_{-}$ & $\mathbf{I}^{+}$ \\
\hline Seguridad Social & 93,8 & 91,3 & 94,4 & 93,7 & 88,6 & 94,8 \\
\hline $\begin{array}{l}\text { Mutualidades del Estado aco- } \\
\text { gidas a la Seguridad Social }\end{array}$ & 2,5 & 2,1 & 2,9 & 1,8 & 1,2 & 2,6 \\
\hline $\begin{array}{l}\text { Mutualidades del Estado aco- } \\
\text { gidas a seguro privado }\end{array}$ & 2,0 & 1,7 & 2,4 & 2,7 & 2,0 & 3,7 \\
\hline Seguro médico privado & 6,4 & 5,8 & 7,1 & 5,9 & 4,8 & 7,2 \\
\hline $\begin{array}{l}\text { Seguro médico concertado de } \\
\text { empresa }\end{array}$ & 1,5 & 1,2 & 1,8 & 0,6 & 0,3 & 1,1 \\
\hline Beneficencia & 0,2 & 0,1 & 0,4 & 0,3 & 0,0 & 0,7 \\
\hline Médico privado & 0,5 & 0,3 & 0,7 & 0,1 & 0,0 & 0,4 \\
\hline Iguala médica & 0,1 & 0,0 & 0,2 & 0,2 & 0,1 & 0,6 \\
\hline Total & 107,0 & & & 105,3 & & \\
\hline
\end{tabular}


cia con los resultados de algunas encuestas realizadas en los últimos años que informan de una práctica generalización de la cobertura pública y de un leve retroceso de la cobertura por modalidades privadas (CuAnter, 1989; Ministerior de Sanidad y Consumo, 1993).

Entre las modalidades de cobertura sanitaria de carácter público, la Seguridad Social representa el elemento principal del Sistema Nacional de Salud (93,8 \% de la población adulta cubierta y 93,7\% de la población infantil), con unos intervalos de confianza muy reducidos en torno al valor medio, más en el caso de los adultos $(91,3 \%$ y $94,8 \%$ ) que en el de los niños (88,6 \% y 94, $8 \%$ ). Alrededor de la Seguridad Social se integraría el resto de los regímenes públicos (con un porcentaje inferior al $3 \%$, y casi residual en el caso de la Beneficencia, en ambas poblaciones). Como es lógico suponer, en estas modalidades poco extendidas los márgenes de confianza oscilan bastante. En todas las modalidades, a excepción de las Mutualidades del Estado acogidas a Seguro Médico Privado, la población infantil presenta unas proporciones de cobertura ligeramente inferiores a las de la población adulta.

Teniendo en cuenta las cifras absolutas de población para 1991 (Instituto Nacional de Estadística, 1992), esto significa que entre 28,6 y 29,5 millones de adultos y entre 6,7 y 7,2 millones de menores de 16 años se benefician de una cobertura por parte de la Seguridad Social, mientras que menos de 90.000 personas en total están protegidos por la Beneficiencia.

Por su parte, el Seguro Médico Privado es la principal modalidad entre las de cobertura privada, cubriendo a un $6,4 \%$ de los adultos, con un intervalo de confianza de entre $5,8 \%$ y 7,1 \%, (es decir, entre 1,8 y 2,2 millones) y a un $5,9 \%$ de los niños, con un intervalo de entre $4,8 \%$ y $7,2 \%$ (entre 360.000 y 540.000 niños) (Cuadro I). Al igual que con las modalidades de carácter público, la población infantil presenta unos niveles porcentuales de cobertura sanitaria privada ligeramente menores que la población adulta. En lo que respecta al Seguro Médico Concertado de Empresa éste ofrece cobertura al 1,5\% de los adultos y al 0,6\% de los niños, con unos intervalos relativamente ajustados a su valor medio (1,2\% y 1,8\%) en aquéllos, lo que representa entre 380.000 y 570.000 personas, siendo mayor su variación en los menores de 16 años reflejando una cobertura de entre 20.000 y 80.000 individuos. 


\section{Cobertura sanitaria exclusiva}

Como era de suponer, el análisis de la cobertura sanitaria exclusiva significa, de hecho, referirse a la realidad anterior, aunque desprovista del aditamiento de tener solapamientos dobles o múltiples, lo que facilita la equiparación entre cobertura en una única modalidad y el uso de servicios sanitarios que pueden tener el mismo carácter de exclusividad entre los detentadores de una determinada modalidad de cobertura.

Hay varios hechos propios de la cobertura exclusiva (Cuadro II). En primer lugar, han desaparecido de la distribución total de modalidades los solapamientos, lo que permite comprobar que existe una conciencia apreciable entre la población de que la generalización de la cobertura sanitaria pública no es una condición que limite el uso simultáneo de otras modalidades. $\mathrm{O}$ dicho de otra manera, existe una práctica común para compaginar dos o más modalidades, realizando un uso diferenciado de ellas según intereses particulares, que no

CUADro II

COBERTURA EXCLUSIVA

\begin{tabular}{l|c|c|c|c|c|c}
\hline \multicolumn{1}{c|}{ Modalidades } & $\begin{array}{c}\text { Pob. } \\
\text { adulta } \\
\mathbf{( \% )}\end{array}$ & $\mathbf{I}_{-}$ & $\mathbf{I}^{+}$ & $\begin{array}{c}\text { Pob. } \\
\text { infantil } \\
\mathbf{( \% )}\end{array}$ & $\mathbf{I}_{-}$ & $\mathbf{I}^{+}$ \\
\hline Seguridad Social & 86,3 & 83,9 & 87,2 & 87,7 & 82,8 & 89,3 \\
\hline $\begin{array}{l}\text { Mutualidades del Estado aco- } \\
\text { gidas a la Seguridad Social }\end{array}$ & 1,9 & 1,6 & 2,3 & 1,4 & 0,9 & 2,2 \\
\hline $\begin{array}{l}\text { Mutualidades del Estado aco- } \\
\text { gidas a seguro privado }\end{array}$ & 1,3 & 1,0 & 1,6 & 2,0 & 1,4 & 2,9 \\
\hline Seguro médico privado & 1,3 & 1,0 & 1,6 & 1,5 & 1,0 & 2,2 \\
\hline $\begin{array}{l}\text { Seguro médico concertado de } \\
\text { empresa }\end{array}$ & 0,3 & 0,2 & 0,5 & 0,2 & 0,0 & 0,5 \\
\hline Beneficencia & 0,1 & 0,1 & 0,2 & 0,2 & 0,1 & 0,7 \\
\hline Médico privado & 0,2 & 0,1 & 0,3 & 0,1 & 0,0 & 0,4 \\
\hline Iguala médica & 0,03 & 0,0 & 0,1 & 0,04 & 0,0 & 0,4 \\
\hline \multicolumn{1}{c}{ Total } & 91,4 & & & 93,2 & & \\
\hline
\end{tabular}


siempre tienen que ver con la oferta de servicios sanitarios de cada una.

En segundo lugar, la dicotomía entre las modalidades públicas y privadas, anteriormente indicada en relación con la cobertura total, no sólo se mantiene sino que se acentúa, no tanto por el descenso de la población cubierta exclusivamente por modalidades de carácter público (que de hecho se produce), sino, sobre todo, por la escasísima representación de la población que detenta, también exclusivamente, modalidades de cobertura de tipo privado.

En efecto, alrededor del $90 \%$ de la población adulta tiene alguna modalidad de cobertura pública, siendo el $86 \%$ de cobertura exclusiva por la Seguridad Social, con un intervalo de confianza bien ajustado al valor medio como consecuencia de su carácter universal $(83,9 \%$ y $87,2 \%$ ), lo que afecta entre 26,3 y 27,3 millones de adultos. En cambio, sólo el 1,8\% puede ser considerado como cubierto exclusivamente por una modalidad privada (no llegan a 600.000 personas, de las que 400.000 son detentadoras de Seguros Médicos Privados con carácter exclusivo). Entre los niños el esquema es muy parecido, con la única salvedad de que la cobertura exclusiva de la Seguridad Social es más elevada $(87,7 \%)$ y con una mayor variación en su intervalo de confianza que entre los adultos. Ello representa que entre 6,2 y 6,7 millones de niños estén cubiertos en exclusiva por la Seguridad Social.

En tercer lugar, destaca notablemente la diferencia observada entre la cobertura exclusiva y total cuando se analiza la población bajo modalidades privadas. De nuevo, es el Seguro Médico Privado la modalidad más destacada entre las de carácter privado (1,3\% de la población adulta y 1,5\% de la infantil), con una variación muy acusada entre los límites inferior y superior de confianza. Esta modalidad ha reducido su porcentaje al ser evaluada como una modalidad en exclusiva, en relación con los datos obtenidos a partir de la cobertura total (6,40\% entre los adultos y de 5,90\% entre los niños). Sin duda, el comportamiento más habitual entre los asegurados de carácter privado es tener, a su vez, como cobertura pública obligatoria la Seguridad Social. En definitiva, sólo entre 310.000 y 500.000 adultos españoles consideran como mejor opción tener una cobertura exclusiva con un Seguro Médico Privado.

En cuarto lugar, el porcentaje de personas que declaran en exclusiva no tener ningún seguro, utilizando por ello la Beneficencia públi- 
ca, el Médico Privado (pagando cada acto médico) o la Iguala Médica, es enormemente reducido $(0,3 \%$ entre los adultos y $0,4 \%$ entre los niños), lo que representa cifras medias de poco más de $100.000 \mathrm{y}$ 35.000 personas, respectivamente.

\section{Solapamientos en la cobertura sanitaria}

Por solapamiento se entiende la situación general en la que un individuo disfruta de 2 o más modalidades de cobertura, de forma simultánea.

Un análisis de los solapamientos de cobertura sirve, entonces, para detectar la situaciones de multiplicidad más comunes en las dos submuestras poblacionales, ya sean adultos, distinguiendo entre titulares y beneficiarios (Cuadro III, Cuadro IV y Cuadro V), o niños en su conjunto (Cuadro VI) ${ }^{1}$.

En lo que respecta a la población adulta titular (Cuadro III), destaca que el mayor volumen de individuos con cobertura solapada se concentra en la Seguridad Social y en los Seguros Médicos Privados, aunque sólo representa el 7,7 \% de la población cubierta por aquella modalidad contra el $70,2 \%$ de la cubierta por estos últimos. Ello se explica, tanto por el carácter universal de la cobertura de la Seguridad Social como por el carácter complementario que detentan los seguros privados con respecto a la asistencia pública.

La complementariedad de estas dos modalidades se comprueba con el hecho de que más de $2 / 3$ partes de los solapamientos con la Seguridad Social (950 personas) proceden de individuos que detentan, a su vez, un Seguro Médico Privado (670 personas). En el mismo sentido, de entre los individuos cubiertos por un Seguro Médico Privado y que tienen alguna otra modalidad de cobertura (681 personas), el $98 \%$ provienen de la Seguridad Social (670 personas). Estos resultados afianzan, por otra parte, una tendencia ya apuntada en años anteriores (Jimeno Martínez, V., 1982; Costas, E., 1987).

El resto de los solapamientos, con y entre otras modalidades, presentan cifras absolutas de menor significación (317 individuos, el

1 Los porcentajes (filas en los cuadros) hacen referencia al número de individuos con modalidades solapadas en relación a la población cubierta por cada modalidad. 
Cuadro III

SOLAPAMIENTOS DE COBERTURA SANITARIA... POBLACIÓN ADULTA (TITULARES)

\begin{tabular}{|c|c|c|c|c|c|c|c|c|c|c|}
\hline \multirow{2}{*}{\multicolumn{2}{|c|}{$\begin{array}{c}\text { Modalidades } \\
\text { población } \\
\text { cubierta } \\
(\mathrm{n}=14.167)\end{array}$}} & \multicolumn{9}{|c|}{ Solapamientos (\%) } \\
\hline & & SS & MSS & MSP & SMP & SCE & $\mathrm{BEN}$ & $\mathrm{MP}$ & IGU & $\begin{array}{l}\text { TOTAL } \\
\text { (ABS.) }\end{array}$ \\
\hline SS & 12.284 & - & 0,28 & 0,48 & 5,45 & 1,35 & 0,02 & 0,09 & 0,06 & $\begin{array}{r}7,73 \\
(950)\end{array}$ \\
\hline MSS & 344 & 9,88 & - & 0,87 & 1,74 & 0,29 & 0,00 & 0,87 & 0,00 & $\begin{array}{c}13,66 \\
(47)\end{array}$ \\
\hline MSP & 245 & 24,08 & 1,22 & - & 2,04 & 0,00 & 0,00 & 0,41 & 0,00 & $\begin{array}{c}27,76 \\
(68)\end{array}$ \\
\hline SMP & 970 & 69,07 & 0,62 & 0,52 & - & 0,00 & 0,00 & 0,00 & 0,00 & $\begin{array}{l}70,21 \\
(681)\end{array}$ \\
\hline $\mathrm{SCE}$ & 232 & 71,55 & 0,43 & 0,00 & 0,00 & - & 0,00 & 0,00 & 0,00 & $\begin{array}{l}71,98 \\
(167)\end{array}$ \\
\hline BEN & 25 & 12,00 & 0,00 & 0,00 & 0,00 & 0,00 & - & 0,00 & 0,00 & $\begin{array}{c}12,00 \\
(3)\end{array}$ \\
\hline MP & 51 & 21,57 & 5,88 & 1,96 & 0,00 & 0,00 & 0,00 & - & 0,00 & $\begin{array}{c}29,41 \\
(15)\end{array}$ \\
\hline IGU & 16 & 43,75 & 0,00 & 0,00 & 0,00 & 0,00 & 0,00 & 0,00 & - & $\begin{array}{c}43,75 \\
(7)\end{array}$ \\
\hline
\end{tabular}

$16 \%$ del conjunto de los solapamientos) que, sin embargo, pueden resultar en proporciones elevadas al ser inferior la población cubierta por éstas (especialmente en los casos de Beneficencia e Igualas Médicas). Tomando entonces las modalidades con mayores valores, ambas Mutualidades y los Seguros Concertados de Empresa, se puede comprobar que sus coberturas se solapan general y lógicamente con la de la Seguridad Social. Además, en el caso de Mutualidades Acogidas a la Seguridad Social se detecta un solapamiento relativamente importante $(12,8 \%$ de sus duplicidades) con el Seguro Médico Privado, en la línea de la complementariedad cobertura pública-privada comentada más arriba. Destaca, por último, que quienes declaran no tener seguro y utilizar siempre Médicos Privados tienden a presentar 
Cuadro IV

SOLAPAMIENTOS DE COBERTURA SANITARIA... POBLACIÓN ADULTA (BENEFICIARIOS)

\begin{tabular}{|c|c|c|c|c|c|c|c|c|c|c|}
\hline \multirow{2}{*}{\multicolumn{2}{|c|}{$\begin{array}{l}\text { Modalidades } \\
\text { población } \\
\text { cubierta } \\
(\mathrm{n}=8.698)\end{array}$}} & \multicolumn{9}{|c|}{ Solapamientos (\%) } \\
\hline & & SS & MSS & MSP & SMP & $\mathrm{SCE}$ & BEN & MP & IGU & $\begin{array}{l}\text { TOTAL } \\
\text { (ABS.) }\end{array}$ \\
\hline SS & 7.770 & - & 0,27 & 0,33 & 2,84 & 0,60 & 0,03 & 0,01 & 0,10 & $\begin{array}{r}4,20 \\
(326)\end{array}$ \\
\hline MSS & 183 & 11,48 & - & 3,28 & 1,09 & 2,73 & 1,64 & 2,73 & 2,19 & $\begin{array}{c}25,14 \\
(46)\end{array}$ \\
\hline MSP & 169 & 15,38 & 3,55 & - & 0,00 & 0,00 & 0,59 & 4,73 & 0,00 & $\begin{array}{c}24,26 \\
(41)\end{array}$ \\
\hline SMP & 392 & 56,38 & 0,51 & 0,00 & - & 0,00 & 0,26 & 2,30 & 0,00 & $\begin{array}{l}59,44 \\
(233)\end{array}$ \\
\hline SCE & 96 & 48,96 & 5,21 & 0,00 & 0,00 & - & 0,00 & 8,33 & 0,00 & $\begin{array}{c}62,50 \\
(60)\end{array}$ \\
\hline BEN & 20 & 10,00 & 15,00 & 5,00 & 5,00 & 0,00 & - & 55,00 & 0,00 & $\begin{array}{c}90,00 \\
(18)\end{array}$ \\
\hline MP & 52 & 1,92 & 9,62 & 15,38 & 17,31 & 15,38 & 21,15 & - & 0,00 & $\begin{array}{l}80,77 \\
(42)\end{array}$ \\
\hline IGU & 16 & 50,00 & 25,00 & 0,00 & 0,00 & 0,00 & 0,00 & 0,00 & - & $\begin{array}{c}75,00 \\
(12)\end{array}$ \\
\hline
\end{tabular}

solapamientos, cuando ello ocurre, casi exclusivamente con la Seguridad Social.

Por su parte, la población beneficiaria adulta (Cuadro IV) presenta un modelo de solapamientos entre modalidades de cobertura bastante similar a la población con titularidad, con la salvedad de la reducción en su volumen debido a que la condición de beneficiario requiere ineludiblemente la existencia de un titular que otorgue cobertura mientras que todo titular no concede, de forma necesaria, la condición de beneficiario. Además, mientras que la cobertura pública se generaliza a toda la población (sin distinguir titulares y beneficiarios en cuanto a coste adicional), la de carácter privado relaciona el precio de la prima con el número de personas cubiertas. 
CuAdro V

SOLAPAMIENTOS DE COBERTURA SANITARIA... POBLACIÓN ADULTA TOTAL: TITULARES + BENEFICIARIOS

\begin{tabular}{|c|c|c|c|c|c|c|c|c|c|c|}
\hline \multirow{2}{*}{\multicolumn{2}{|c|}{$\begin{array}{l}\text { Modalidades } \\
\text { población } \\
\text { cubierta } \\
(n=25.964)\end{array}$}} & \multicolumn{9}{|c|}{ Solapamientos (\%) } \\
\hline & & SS & MSS & MSP & SMP & SCE & BEN & MP & IGU & $\begin{array}{l}\text { TOTAL } \\
\text { (ABS.) }\end{array}$ \\
\hline SS & 21.382 & - & 0,49 & 0,60 & 4,94 & 1,16 & 0,09 & 0,31 & 0,11 & $\begin{array}{c}7,69 \\
(1.645)\end{array}$ \\
\hline MSS & 664 & 15,81 & - & 1,51 & 1,66 & 0,90 & 0,45 & 1,51 & 0,60 & $\begin{array}{l}22,44 \\
(149)\end{array}$ \\
\hline MSP & 564 & 22,70 & 1,77 & - & 1,06 & 0,18 & 0,18 & 1,60 & 0,00 & $\begin{array}{l}27,48 \\
(155)\end{array}$ \\
\hline SMP & 2.415 & 43,73 & 0,46 & 0,25 & - & 0,08 & 0,04 & 0,46 & 0,00 & $\begin{array}{c}45,01 \\
(1.087)\end{array}$ \\
\hline SCE & 579 & 42,66 & 1,04 & 0,17 & 0,35 & - & 0,17 & 2,25 & 0,00 & $\begin{array}{l}46,63 \\
(270)\end{array}$ \\
\hline BEN & 82 & 24,39 & 3,66 & 1,22 & 1,22 & 1,22 & - & 13,41 & 0,00 & $\begin{array}{c}45,12 \\
(37)\end{array}$ \\
\hline MP & 222 & 29,73 & 4,50 & 4,05 & 4,95 & 5,86 & 4,95 & - & 0,00 & $\begin{array}{l}54,05 \\
(120)\end{array}$ \\
\hline IGU & 56 & 41,07 & 7,14 & 0,00 & 0,00 & 0,00 & 0,00 & 0,00 & - & $\begin{array}{c}48,21 \\
(27)\end{array}$ \\
\hline
\end{tabular}

Otro elemento de comparación con la población adulta (Cuadro V) lo constituye la matriz de solapamientos referida a la población infantil (Cuadro VI). En este caso es uno de los padres el que refiere la condición de titular o beneficiario de su hijo en las modalidades investigadas, suponiéndose que, en la mayoría de los casos, los menores de 16 años son beneficiarios.

Los datos de la matriz muestran lo siguiente:

- una proporción de solapamientos (10,4\%) inferior a la población adulta en su conjunto (13,4\%),

- las dos modalidades con mayor volumen de solapamiento son de nuevo la Seguridad Social y los Seguros Médicos Privados, que 
Cuadro VI

SOLAPAMIENTOS DE COBERTURA SANITARIA... POBLACIÓN INFANTIL TITULARES + BENEFICIARIOS

\begin{tabular}{|c|c|c|c|c|c|c|c|c|c|c|}
\hline \multirow{2}{*}{\multicolumn{2}{|c|}{$\begin{array}{c}\text { Modalidades } \\
\text { población } \\
\text { cubierta } \\
(\mathrm{n}=6.116)\end{array}$}} & \multicolumn{9}{|c|}{ Solapamientos (\%) } \\
\hline & & SS & MSS & MSP & SMP & SCE & BEN & $\mathrm{MP}$ & IGU & $\begin{array}{l}\text { TOTAL } \\
\text { (ABS.) }\end{array}$ \\
\hline SS & 5.248 & - & 0,32 & 0,63 & 4,46 & 0,38 & 0,02 & 0,04 & 0,13 & $\begin{array}{r}5,98 \\
(314)\end{array}$ \\
\hline MSS & 112 & 15,18 & - & 0,89 & 0,00 & 0,00 & 0,00 & 0,00 & 0,00 & $\begin{array}{c}16,07 \\
(18)\end{array}$ \\
\hline MSP & 182 & 18,13 & 0,55 & - & 0,55 & 1,10 & 0,00 & 0,00 & 0,00 & $\begin{array}{c}20,33 \\
(37)\end{array}$ \\
\hline SMP & 548 & 42,70 & 0,00 & 0,18 & - & 0,00 & 0,00 & 0,00 & 0,00 & $\begin{array}{l}42,88 \\
(235)\end{array}$ \\
\hline $\mathrm{SCE}$ & 52 & 38,46 & 0,00 & 3,85 & 0,00 & - & 0,00 & 0,00 & 0,00 & $\begin{array}{c}42,31 \\
(22)\end{array}$ \\
\hline BEN & 15 & 6,67 & 0,00 & 0,00 & 0,00 & 0,00 & - & 0,00 & 0,00 & $\begin{array}{l}6,67 \\
\text { (1) }\end{array}$ \\
\hline $\mathrm{MP}$ & 8 & 25,00 & 0,00 & 0,00 & 0,00 & 0,00 & 0,00 & - & 0,00 & $\begin{array}{c}25,00 \\
(2)\end{array}$ \\
\hline IGU & 16 & 43,75 & 0,00 & 0,00 & 0,00 & 0,00 & 0,00 & 0,00 & - & $\begin{array}{c}43,75 \\
(7)\end{array}$ \\
\hline
\end{tabular}

suponen, en su totalidad, el $36 \%$ de los solapamientos de cobertura en población infantil contra el $30 \%$ en población adulta,

- las proporciones de duplicidad de cobertura entre Seguridad Social y Seguros Privados son superiores entre los niños: el $75 \%$ de los solapamientos detectados en la Seguridad Social proceden de aquéllos que tienen a su vez un Seguro Médico Privado y casi el $100 \%$ de los solapamientos de esta última modalidad provienen de aquélla. Estas cifras, entre la población adulta en su conjunto, son el $64 \%$ y el $97 \%$, respectivamente,

- una práctica inexistente de solapamientos entre el resto de las modalidades que ya suponían cifras muy reducidas entre la población adulta total. 
Distribución geográfica de la cobertura sanitaria

El análisis de la distribución geográfica de la cobertura sanitaria debe tener en cuenta varias premisas:

a) la significación del cuestionario dirigido a la población infantil es a escala nacional y no de comunidad autónoma, salvo excepciones, por lo que se hurta la posibilidad de comparación territorial de la coberturas (total y exclusiva) entre esta población y la adulta,

b) el hecho de la universalización de la cobertura pública minimiza las diferencias entre comunidades con este carácter,

c) los valores tan rducidos para el resto de las modalidades, a excepción de los Seguros Médicos Privados, limitan las posibilidades de su comparación ante la aleatoriedad que puede derivarse de ello.

Por estos motivos, sólo se estudia la distribución geográfica de las modalidades de la Seguridad Social y Seguros Médicos Privados para la población adulta, en su cobertura total y exclusiva.

En primer lugar, no parece definirse un modelo geográfico claro en la distribución de la cobertura total por la Seguridad Social (Mapa 1). Los valores en torno o inferiores a la medida se sitúan en las franjas norte (Asturias, Cantabria, País Vasco, Navarra y Aragón) y sur (Extremadura, Andalucía y Murcia), a las que se añaden Madrid y Baleares, como casos particulares. Sin embargo, cuando se comparan estos resultados con los de cobertura exclusiva por esta modalidad, sí se confirma, entonces, un modelo territorial: valores superiores a la media generalizados en el conjunto de las comunidades, salvo en Madrid, Aragón, Cataluña y Baleares. Los solapamientos contemplados en la cobertura total pueden ser la causa de estas diferencias, dentro de la tendencia hacia la universalización de la cobertura.

En segundo lugar y por lo que respecta a la cobertura total por Seguros Médicos Privados (Mapa 2), el modelo geográfico resultante complementa al anterior: los valores inferiores o en torno a la media se generalizan para el conjunto de las comunidades autónomas, a excepción, de nuevo, de Madrid, Cataluña y Baleares. Los datos sobre cobertura exclusiva por esta modalidad matizan este modelo: Madrid y Baleares se confirman como comunidades con elevadas proporciones, a las que se añadirían País Vasco, Navarra, Aragón y Murcia, quedando, sin embargo, Cataluña con un valor en torno a la media.

$$
-389-
$$


MAPA 1. SEGURIDAD SOCIAL
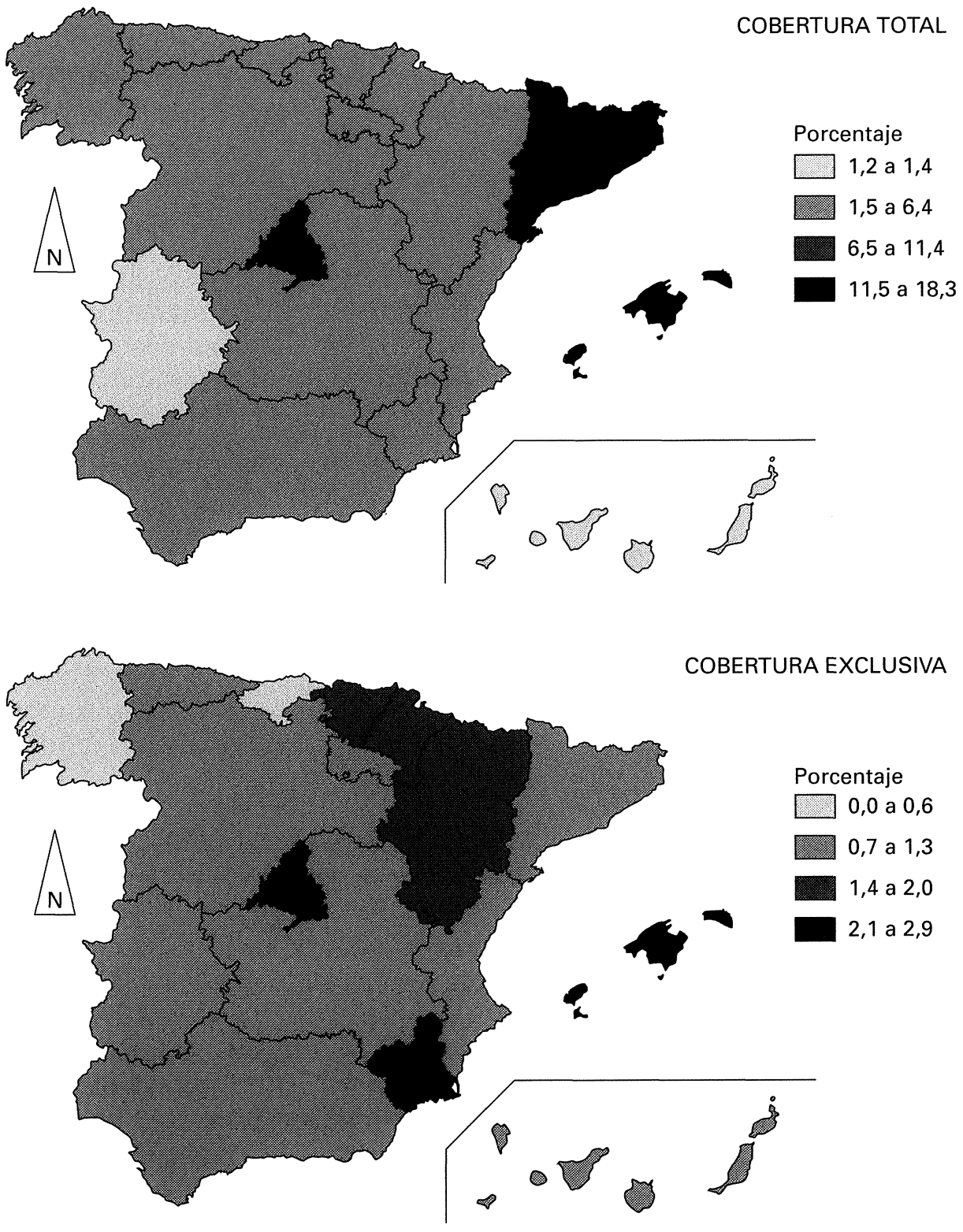
MAPA 2. SEGUROS MÉDICOS PRIVADOS
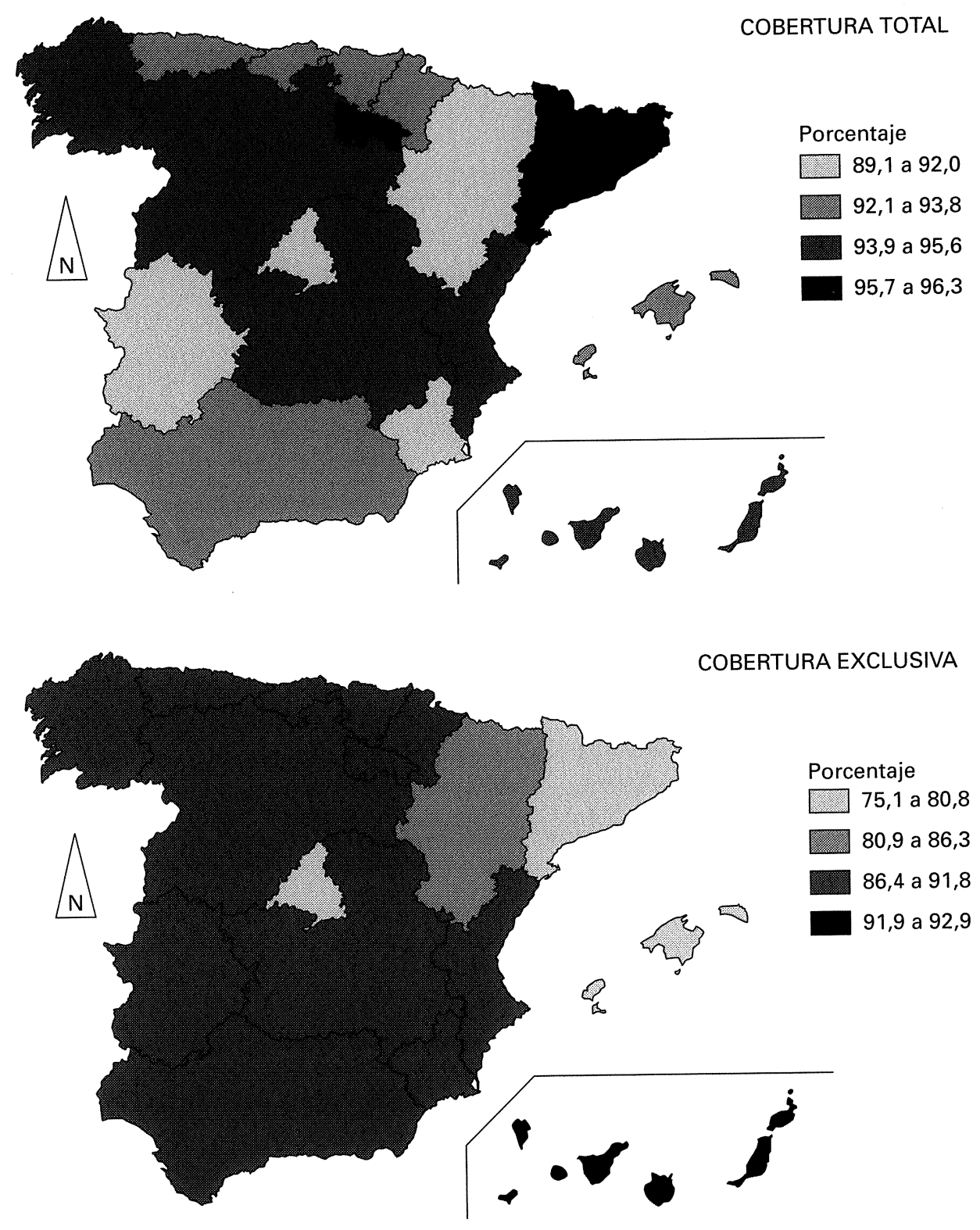
La extensión de la cobertura por Seguro Médico Privado básicamente en regiones de una economía urbana y desarrollada se corresponde con unos factores que se han demostrado influyentes en ello. La decisión de toma de un seguro privado estaría relacionada con:

- rentas relativamente más elevadas que determinan una mayor capacidad de compra familiar (Murillo, C., 1992; Murillo, C., Calonge, S. y González, Y., 1992).

- la situación profesional del cabeza de familia (p. e. inercia histórica de los trabajadores autónomos a detentar este tipo de cobertura ya que hasta 1984 no estuvieron cubiertos por la Seguridad Social) (Murillo, C., 1992; Murillo, C., Calonge, S. y González, Y., 1992),

- factores de tipo perceptivo o de opinión sobre la situación actual de la sanidad pública en relación con la privada, opiniones que pueden estar condicionadas, también, por la mayor implantación en determinadas regiones de entidades privadas proveedoras de asistencia sanitaria (p. e. en Madrid, Barcelona y Zaragoza tienen su sede algunas de estas entidades) (CuAnter, 1989; Murillo, C., 1992),

- componentes demográficos, como la estructura por edad y sexo o la composición familiar, que, indirectamente, tienden a condicionar los anteriores factores.

\section{Conclusiones}

Los dos caracteres esenciales de la cobertura sanitaria de la población española son la universalización de la sanidad pública y la complementariedad, con respecto a ésta, de la sanidad privada. Aunque la cifra pueda parecer pequeña, no es despreciable que casi el $8 \%$ de la población adulta cubierta por la Seguridad Social tenga otra cobertura sanitaria, básicamente Seguros Médicos Privados. A su vez, el 98\% de la población con Seguros Privados está cubierta por la Seguridad Social. Entre la población infantil, el volumen de solapamientos es inferior pero las duplicidades de cobertura entre la Seguridad Social y los Seguros Médicos Privados son mayores. 
NIVELES Y DISTRIBUCIÓN GEOGRÁFICA DE LA COBERTURA SANITARIA...

Los hechos que influyen sobre el recurso simultáneo a ambas coberturas podrían resumirse a grandes rasgos en:

- una mejora de los niveles socioeconómicos y estándares de vida de estratos de la población más amplios que exigen una mayor calidad de la asistencia sanitaria (en lo que atañe a masificación, listas de espera, elección de facultativos...) (Murillo, C., 1992),

- un relativo estancamiento de la calidad de la oferta sanitaria pública, en los conceptos anteriormente expuestos, derivado de la universalización de la cobertura cuando no se proveen los recursos necesarios para su mejora (Murillo, C., 1992; Costas, E., 1987),

- una opinión y percepción de los consumidores de servicios sanitarios sobre las deficiencias de la sanidad pública (fundamentalmente en lo que se refiere a tiempos de espera, escasa duración de la consulta, hostelería, trato recibido) como valor más positivo a considerar en la elección de cobertura privada (CUANTER, 1989).

En definitiva, es un fenómeno complejo en el que a los factores objetivos de oferta y demanda se suman factores subjetivos de percepción de la realidad sanitaria, no siempre libres de esquemas ideológicos y de polémicas en torno a grupos profesionales y sociales, interesados en modelos sanitarios distintos. En este contexto se perfila un debate a propósito de cuáles deberían ser las relaciones entre financiación, provisión y gestión de recursos (Rovira, J., 1987; Vivas, D. y Sánchez Ruano, E., 1993) para una mejora de la asistencia sanitaria, en sus aspectos de equidad, eficacia y eficiencia (Puig, J., 1987; Rodríguez, M., Calonge, S. y Reñe, J., 1992). En esta línea, la propia administración pública reflexionó sobre estas (y otras) cuestiones dentro de un objetivo general de reforma del Sistema Nacional de Salud (Comisión de Análisis y Evaluación del Sistema Nacional de Salud, 1991), iniciativa que dio lugar a diversas apreciaciones sobre las medidas recomendadas (López I Casasnovas, G., 1991; Asociación de Economía de la Salud, 1992; Ventura, J., 1992a; Ventura, J., 1992b). 


\section{BIBLIOGRAFÍA}

Asociación de Economía de la SAlud (1992): «Situación actual del Sistema Nacional de Salud y propuestas de mejora». El Sistema Nacional de Salud de los 90 (Estrategia de salud, planificación y mercado), XI Jornadas de Economía de la Salud, Asociación Española de Economía de la Salud, Valencia, p. 633-656.

Comisión de Análisis y Evaluación del Sistema Nacional de Salud (1991). Informe y Recomendaciones (Conclusiones y síntesis final a los 12 volúmenes que recogen informes de subcomisiones, entidades, organismos y asesores), Madrid, $79 \mathrm{p}$.

Costas, E. (1987): «Calidad de la asistencia y sanitaria pública». Sistema Nacional de Salud. La reforma sanitaria. SESPAS, Madrid, p. 129-136.

CUANTER (1989): Estudio de la población española sobre la percepción de la asistencia sanitaria.

Ley 14/1986, del 25 de abril, General de Sanidad, Cap. I, Art. 3.2. (B.O.E. 102, de 29 de abril).

Instituto Nacional de Estadística (1992): Censos de Población y Viviendas 1991. Muestra avance. Principales resultados. INE, Madrid, $223 \mathrm{p}$.

Jimeno Martínez, V. (1982): «Desigualdades en las atenciones sanitarias de España». Revista Internacional de Sociologia, núm. 42, abril-junio, tomo XL, p. 183-206.

López I CASAsnovas, G. (1991): «El Informe "Abril” de reforma de la asistencia sanitaria». Papeles de Economia Española, núm. 48, p. 99-105.

Ministerio de Sanidad y Consumo (1987): Encuesta de Cobertura Sanitaria.

Ministerio de Sanidad y Consumo (1993): Barómetro Sanitario.

MuriLlo, C. (1992): «La industria del seguro privado y los seguros sanitarios». Revista de Economía, 13, p. 84-90

Murillo, C.; Calonge, S. y GonzAlez, Y. (1992): «Los seguros privados de asistencia sanitaria». El Sistema Nacional de Salud de los 90 (Estrategia de salud, planificación y mercado), XI Jornadas de Economía de la Salud, Asociación Española de Economía de la Salud, Valencia, p. 419-427.

Puig, J. (1987): «Equidad en la asignación territorial de recursos financieros versus equidad en el sistema sanitario». Sistema Nacional de Salud. La reforma sanitaria. SESPAS, Madrid, p. 45-61.

Rodríguez, M.; Calonge, S. Y Reñe, J. (1992): «Análisis de la (in)equidad en la financiación y la provisión de los servicios en el sistema sanitario español». El Sistema Nacional de Salud de los 90 (Estrategias de salud, planificación y mercado), XI Jornadas de Economía de la Salud, Asociación Española de Economía de la Salud, Valencia, pp. 543-562.

Rodríguez, V.; Fernández-Mayoralas, G.; Pumares, P. y Barrios, L. (1994): Análisis de las modalidades de cobertura sanitaria de la población española según la Encuesta Nacional de Salud, 1993. Dirección General de Aseguramiento y Planificación Sanitaria. Ministerio de Sanidad y Consumo. Inédito. 2 volúmenes.

RoviRA, J. (1987): «Alternativas de provisión pública y privada en un sistema de financiación pública». Sistema Nacional de Salud. La reforma sanitaria. SESPAS, Madrid, pp. 167-183.

VentuRA, J. (1992a): «La reforma del servicio nacional de salud (El Informe Abril)». Información Comercial Española, núms. 708-709, pp. 53-61.

Ventura, J. (1992b): «¿Competencia o cooperación en sanidad?». Revista de Economía, núm. 11 , pp. 46-50.

Vivas, D. y SÁnchez Ruano, E. (1993): Informe Delphi: el Sistema Sanitario Español. Estrategias, tendencias y áreas de desarrollo. Valencia, MCQ Ediciones, $52 \mathrm{p}$.

World Health Organization (1981): Health Services in Europe. Who. Regional Office for Europe, 3rd. ed., 2 vols. Copenhagen.

World Health ORganization (1991): Organization and Financing of Health Care Reform in Countries of Central and Eastern Europe. Who, Geneva, 44 p. 


\section{NIVELES Y DISTRIBUCIÓN GEOGRÁFICA DE LA COBERTURA SANITARIA EN ESPAÑA}

Resumen: Tomando como información la Encuesta Nacional de Salud de 1993, en este artículo se analizan los niveles de cobertura sanitaria de la población española, distinguiendo entre modalidades (públicas y privadas) y tipo de cobertura (total, exclusiva y solapamiento). Se identifican, además, los modelos geográficos que se perfilan en la distribución de las modalidades más extendidas.

Niveles y distribución de cobertura sanitaria responden a los dos rasgos fundamentales del sistema de salud español: la práctica universalización de la cobertura sanitaria pública, básicamente a través de la modalidad de Seguridad Social, y el carácter complementario de la cobertura privada, mediante los Seguros Médicos Privados.

Palabras clave: Sistema Nacional de Salud. Cobertura Sanitaria. Encuesta Nacional de Salud.

\section{HEALTH COVER IN SPAIN. LEVELS AND GEOGRAPHIC DISTRIBUTION}

ABsTRACT: From the National Health Survey of Spain, the health cover of the population is analysed in this paper according to public or private insurance and kind of cover: total, exclusive or multiple.

Levels and geographical distribution of health cover confirm that the public financing system covers the entire population and the private insurance is a complementary element of the Spanish health system.

KEY words: National Health System. Health Cover. National Health Survey.

NIVEAUX DE PROTECTION ET DISTRIBUTION GEOGRAPHIQUE DE L'ASSURANCE MALADIE EN ESPAGNE

Résume: Dans cet article, basé sur les données de l'Enquête Nationale sur la Santé faite en 1993, on analyse les niveaux de l'assurance maladie de la population espagnole en faisant la différence entre les modalités (publiques et privées) et les types de couverture (totale, exclusive et multiple). En outre, on identifie les modèles géographiques qui se dessinent dans la distribution des modalités les plus développées.

Les niveaux et la distribution de l'assurance maladie repondent à deux caracteristiques fondamentales du Système d'Assurance Maladie espagnol: l'une correspond à la presque généralisation de l'assurance maladie publique à travers la Sécurité Sociale et l'autre à l'Ássurance Maladie des régimes privés comme éléments complémentaires du système.

Mots CLÉ: Système National d'Assurance Maladie. Assurance Maladie. Enquête Nationale sur la Santé 